\title{
Comment on "Estimating the Bioconcentration Factors of Hydrophobic Organic Compounds from Biotransformation Rates Using Rainbow Trout Hepatocytes" by Trowell et al. in AECT
}

\author{
Kai-Uwe Goss ${ }^{1,2}$
}

Received: 3 May 2018 / Accepted: 14 August 2018 / Published online: 4 September 2018

(c) Springer Science+Business Media, LLC, part of Springer Nature 2018

In their recent paper, Trowell et al. performed an in vitro in vivo extrapolation (IVIVE) of metabolic rate constants to better assess bioconcentration in fish. I had difficulties reproducing the formulas that are used and putting them in context and would like to ask for clarifications.

It appears that the extrapolation formula presented is not equivalent to the extrapolation formula that was published by the same group a few months ago (Lee et al. 2017). A discussion of this discrepancy would be helpful. In fact, both variants differ from the approach by Nichols et al. (2013), which has so far widely been used in IVIVE for fish. This discrepancy to the Nichols approach remains if one removes the flow limitation in the Nichols approach and if one ignores the different approaches to estimate unbound fractions. Again, a discussion would be helpful.

In terms of the units, one finds that $\mathrm{k}_{\mathrm{r}}$ and $\mathrm{k}_{\mathrm{MET}}$ in the paper by Trowell et al. have not the expected units. The liver reaction rate constant $\mathrm{k}_{\mathrm{r}}$ is supposed to have the unit $1 / \mathrm{min}$, but if one inserts all units in Eq. 2 for $\mathrm{k}_{\mathrm{r}}$ :
The volume of the incubation medium cannot be canceled against the volume of liver.

Another multiplication with the cell density of hepatocytes is performed in Eq. 3 (in addition to the multiplication with the same factor in Eq. 2). It does not seem to be plausible that the square of the hepatocyte cell density should be required when extrapolating from the in vitro rate constant, $\mathrm{k}_{\mathrm{d}}$, to the in vivo rate constant by combining Eqs. 2 and 3 in the paper.

Also, the units of the resulting whole fish biotransformation rate constant, $\mathrm{k}_{\mathrm{MET}}$, are not $1 / \mathrm{min}$ as one would expect (note, $\mathrm{M}_{\mathrm{lo}}$ is defined as "the storage capacity of the liver expressed as a fraction of the chemical storage capacity of the organism" and should thus be dimensionless, just as the quotient of the two unbound fractions in blood and incubation):

$k_{\text {MET }}=\left(\frac{1}{\min }\right) *\left(\frac{m l_{\text {incubation }}}{m l_{\text {liver }}}\right)\left(\frac{g_{\text {liver }}}{m l_{\text {liver }}}\right)$

$k_{r}=k_{d}\left(\frac{1}{\min }\right) * H\left(\frac{\#_{\text {hepatocytes }}}{g_{\text {liver }}}\right) * d_{l}\left(\frac{g_{\text {liver }}}{m l_{\text {liver }}}\right) /\left[C_{h}\left(\frac{g_{\text {hepatocytes }}}{m l_{\text {incubation }}}\right) * d_{c}\left(\frac{\#_{\text {hepatocytes }}}{g_{\text {hepatocytes }}}\right)\right]$

one receives:

$k_{r}=\left(\frac{1}{\min }\right) *\left(\frac{m l_{\text {incubation }}}{m l_{\text {liver }}}\right)$

Kai-Uwe Goss

kai-uwe.goss@ufz.de

1 Department of Analytical Environmental Chemistry, Helmholtz Centre for Environmental Research GmbH - UFZ, Permoserstr. 15, 04318 Leipzig, Germany

2 Institute of Chemistry, University of Halle-Wittenberg, Kurt-Mothes-Str. 2, 06120 Halle, Germany

\section{References}

Lee YS, Lo JC, Otton SV, Moore MM, Kennedy CJ, Gobas F (2017) In vitro to in vivo extrapolation of biotransformation rates for assessing bioaccumulation of hydrophobic organic chemicals in mammals. Environ Toxicol Chem 36:1934-1946

Nichols JW, Huggett DB, Arnot JA, Fitzsimmons PN, Cowan-Ellsberry CE (2013) Toward improved models for predicting bioconcentration of well-metabolized compounds by rainbow trout using measured rates of in vitro intrinsic clearance. Environ Toxicol Chem 32:1611-1622 